\title{
In memoriam Georg Hossli
}

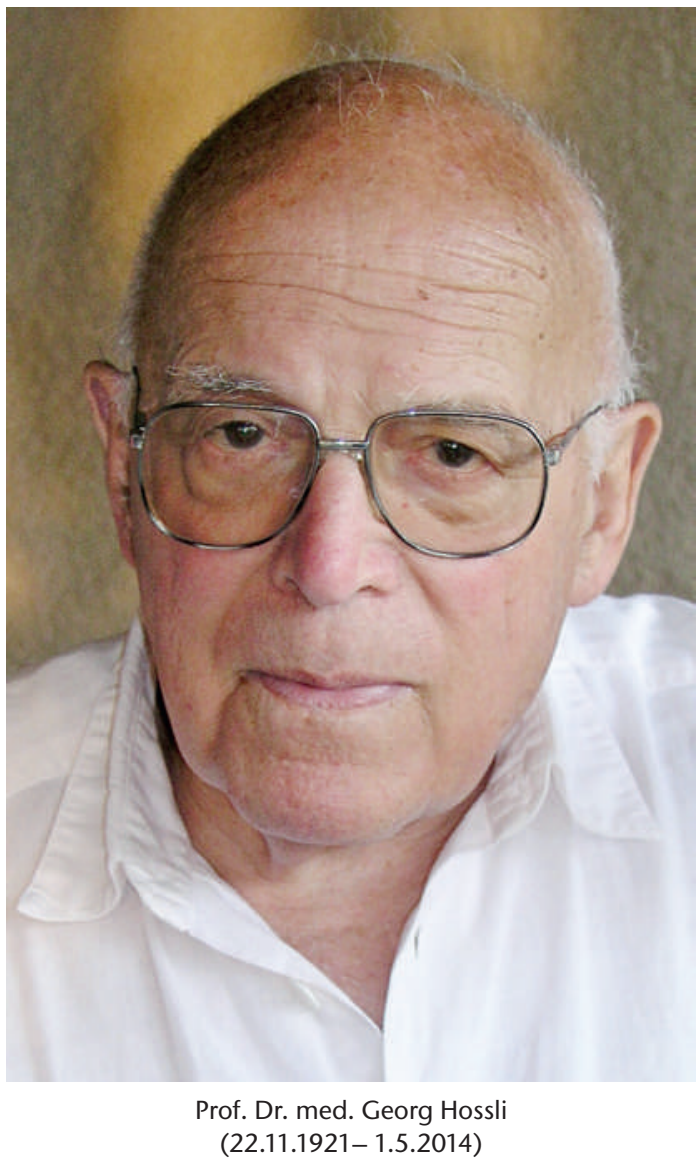

In seinem 93.Lebensjahr starb am vergangenen 1. Mai Prof. Dr. med. Georg Hossli, der erste Ordinarius für Anästhesiologie an der Universität Zürich. Bis zu seinen letzten Tagen blieb sein Geist wach und klar. Noch einmal erfreute er sich am hellen Grün des Frühlings. Seine Gedanken flogen zurück ins heimatliche Fricktal mit den blühenden Wiesen und Bäumen.

Nach dem Staatsexamen und nach Abschluss seiner Dissertation zog es Georg Hossli in die Ferne. Als Schiffsarzt auf einem schwedischen Frachter übte er auf der Überfahrt nach Brasilien eine erste ärztliche Tätigkeit aus.

Es folgte die Assistentenzeit an der Chirurgischen Universitätsklinik Zürich unter Professor Alfred Brunner. Wie üblich hatte er als Jüngster der Ärzteschaft nach dürftiger Einführung Narkosen durchzuführen. Rasch erfasste er nicht nur die technischen und organisatorischen Unzulänglichkeiten dieses wenig geachteten Dienstes, sondern auch das grosse
Entwicklungspotential der Anästhesie als wichtige Voraussetzung für Fortschritte auf allen Gebieten der Chirurgie. Es war die Zeit, da sich, auf wissenschaftlichen Grundlagen abgestützt, das neue Fachgebiet der Anästhesiologie etablierte. Studienreisen führten ihn nach Europa und die USA.

Mit höflicher Bestimmtheit und diplomatisch geschickt artikulierte er visionäre Vorstellungen und setzte sie mit nie ermüdender Beharrlichkeit um. Wer hätte sich damals vorstellen können, dass einmal auf den Dächern von Spitälern Helikopter mit Notfallpatienten landen würden?

Mit Unterstützung weitblickender Fakultätsmitglieder gelang es, 1954 eine zentrale Anästhesieabteilung einzurichten. Georg Hossli wurde zum Leitenden Arzt gewählt und erhielt einen Lehrauftrag für Anästhesiologie, Wiederbelebung und Schockbekämpfung. Harte Aufbauarbeit war gefordert. Stellen im Arzt- und Pflegedienst mussten erkämpft und Schulungskonzepte entworfen werden. Man schätzte ihn als Lehrer und Instruktor. Seine Konzepte waren klar und einprägsam. Er führte durch beispielhaftes Vorbild und stete Einsatzbereitschaft. Seine Kritik war präzis, wohlwollend und nie verletzend. 1960 habilitierte er sich mit einer Arbeit über die Anästhesie bei thorakalen Eingriffen.

1965 wählte der Regierungsrat des Kantons Zürich PD Dr. Georg Hossli zum Extraordinarius und zum Direktor des neu geschaffenen selbständigen «Instituts für Anästhesiologie der Universitätskliniken». Anästhesiologie und Wiederbelebung wurden obligatorische Vorlesungs- und Prüfungsfächer für Medizinstudenten. 1970 erfolgte die Umwandlung des Extraordinariats in ein Ordinariat, nach Basel das zweite in der Schweiz.

Zu einer Zeit, da es noch keine Intensivbehandlungsstationen gab, hat Georg Hossli in der Dauerbeatmung von Starrkrampf- und Kinderlähmungspatienten wegweisende Pionierarbeit geleistet. Schwerpunkte seines Schaffens waren Katastrophenmedizin, Probleme der Wiederbelebung, der Primärversorgung und des Transportes Schwerverletzter und akut lebensbedrohter Erkrankter. Seine zahlreichen Publikationen auf diesem anspruchsvollen Spezialgebiet haben ihm internationalen Ruf sowie 1985 den Auftrag zur Organisation eines Weltkongresses in Zürich eingebracht.

Als Pionier in seinem eigenen Fach hat Georg Hossli auch die Pionierzeiten vieler chirurgischer 
Spezialgebiete am UniversitätsSpital Zürich aktiv miterlebt und durch die Erfüllung der hohen Anforderungen an die Anästhesie mitbeeinflusst und mitgefördert: so zum Beispiel die Herzchirurgie, die Mikrochirurgie in Neuro- und Wiederherstellungschirurgie, die Organtransplantations- und -replantationschirurgie und die interdisziplinäre Rekonstruktionschirurgie der Kieferchirurgie. Der Herausforderung der fortschreitenden Entwicklung dieser Spezialgebiete hat sich Georg Hossli stets souverän gestellt, nicht zuletzt auch vertrauend auf seinen Stab motivierter und kompetenter Mitarbeiter.

Im Rang eines Oberstleutnants der Sanität diente Georg Hossli als Fachdienstchef für Anästhesiologie und Wiederbelebung im Schweizer Armeestab. Mehr als 25 Jahre lang war er auch kompetenter Berater der Schweizerischen Lebensrettungsgesellschaft und des Schweizerischen Samariterbundes. Mit grosser Hingabe hat er beim Aufbau der REGA mitgewirkt. Von 1958, von Anfang an, bis 1986 stellte er seine Dienste als Chefarzt, Notarzt bei vielen Luftrettungen, es sollen gegen 800 gewesen sein, und als ärztlicher Leiter bei Grosseinsätzen zur Verfügung. Sein Funkgerät sicherte seine stete Erreichbarkeit.

Georg Hossli hat in der Ausbildung von Ärzten und Pflegepersonal der Anästhesie in der Schweiz eine führende Rolle gespielt. An der Besetzung von Lehrstühlen und Chefarztstellen auf seinem Gebiet hat er in unserem Lande den grössten Anteil. Das obligatorische Fachexamen zur Erlangung des Titels «Spezialarzt für Anästhesiologie» geht zum grössten Teil auf seine Initiative zurück. Im Kreis seiner Schüler gedenken auch 224 Zahnärzte, die er zu Militäranästhe- sisten ausbildete, seiner in hoher Anerkennung und Dankbarkeit.

Ein letztes Mal leistete er Notfallhilfe im Alter von 88 Jahren. Auf einer Fahrt mit einer Gruppe von Sanitätsoffizieren zu einer Besichtigung von Festungswerken am Gotthard sank der Festungskommandant in sich zusammen, pulslos, lichtstarre Pupillen. Noch einmal führte Georg Hossli. Auf der Strasse neben dem Car reanimierte er den Leblosen, gab Anweisung zur Alarmierung des Rettungshelikopters, defibrillierte und intubierte. Mit stabilem Kreislauf konnte der Kommandant dem koronarchirurgischen Noteingriff zugeführt werden. Er überlebte ohne Schaden und blieb Georg Hossli freundschaftlich verbunden.

Ein Einsatz besonderer Art sei noch erwähnt. Beim Besuch von Papst Johannes Paul II. im Juni 1984 flog ein REGA-Helikopter mit dem Notarzt Hossli die ganze Strecke direkt hinter dem Papsthelikopter. Während des ganzen Papstbesuchs war damit die sofortige Interventionsmöglichkeit gegeben. Der Dank des Papstes war ihm sicher. Diese Episode veranlasste den Pfarrer im Rahmen der schlichten Abdankungsfeier zu sagen, es hätte wohl noch kein anderer Arzt einen richtigen Heiligen beschützt.

Zu seinem 80. Geburtstag schrieb Pius Frey:

«Prof. Georg Hossli hat ein riesiges Werk geleistet. Er wusste, was getan werden musste, wann der rechte Zeitpunkt war, und hat die Aufgaben konsequent durchgeführt. Ihm war der Satz von Hippokrates «Die rechte Zeit ist nur ein Augenblick» ein Leitsatz.»

Ein grosser Arzt und Lehrer, ein guter Freund und lieber Mensch ist von uns gegangen.

Prof. Dr. med. Willy Stoll, Prof. Dr. med. Ruth Gattiker 